\title{
MicroED on lipidic cubic phase embedded crystals
}

\section{B Nannenga ${ }^{1}$ \\ ${ }^{1}$ Arizona State University, Tempe, AZ brent.nannenga@asu.edu}

The lipidic cubic phase (LCP) technique is a proven method to facilitate the growth of high-quality crystals that are otherwise difficult to grow by other methods. Because crystals grown in LCP can be limited in size, improved techniques for structure determination from these small crystals are important. Microcrystal electron diffraction (MicroED) is a technique that uses a cryo-TEM to collect electron diffraction data and determine high-resolution structures from very thin micro and nanocrystals. However, the viscosity of the LCP matrix makes MicroED sample preparation extremely difficult. In this presentation, we will discuss our results and recent progress on using modified LCP and MicroED protocols to analyze crystals embedded in LCP.

Acta Cryst. (2020). A76, a166 